\title{
Safety and Efficacy of Iron Isomaltoside in Two Cases with Liver Cirrhosis and Iron Deficiency Anemia
}

\author{
Liuben Milatchkov, Tanya Petkova, Deian Jelev, Rosen \\ Nikolov, Lyudmila Mateva, Zaharyi Krastev
}

University Hospital "St. Ivan Rilski" Sofia, Clinic of Gastroenterology

\begin{abstract}
Anemia of diverse etiology occurs in about $75 \%$ of patients with liver cirrhosis. Mucosal injury due to portal hypertension is an underestimated cause of occult bleeding and iron-deficiency anemia. Iron isomaltoside is a parenteral agent with low free-iron toxicity that is used as a short-term infusion without need of test-dose. There are no data on its safety and efficacy in patients with liver cirrhosis. Two males (30 and 66 years old) with Child-A liver cirrhosis and iron-deficiency anemia were evaluated. Both of them had pre-treatment hemoglobin level $<90 \mathrm{~g} / \mathrm{l}$. The older patient once was anti-RBC antibody positive once. No obvious signs and no recent history of blood-loss were established. The first patient had severe portal hypertension: large oesophageal varices with red signs and portal hypertensive gastropathy, while the other one was with significant portal colonopathy, hypertensive gastropathy and medium oesophageal varices. Endoscopic evaluation in both patients showed no active bleeding but marked mucosal friability. Other sources of blood-loss were excluded. Anemia had been previously orallytreated with ferric-hydroxide polymaltose complex, however no improvement was achieved. Both patients were treated with iron isomaltoside infusions. The younger one received a single dose of $1000 \mathrm{mg}$ and after one month $\mathrm{Hb}$ levels increased from 80 to $122 \mathrm{~g} / \mathrm{l}$. The older subject was treated initially with $600 \mathrm{mg}$. As a result, $\mathrm{Hb}$ levels remained relatively unchanged. A second infusion in a dose of $1200 \mathrm{mg}$ was given amonth later and elevation of $\mathrm{Hb}$ from 75 to $95 \mathrm{~g} / \mathrm{l}$ was achieved. The ferritin rose from 14 to $23 \mathrm{ng} / \mathrm{mL}$. Treatment was well tolerated and no side effects were observed. This is the first report for treatment of iron-deficiency anemia by iron isomaltoside infusion in cirrhotic patients. Further studies are needed to establish its safety and efficacy in subjects with liver cirrhosis.
\end{abstract}

Keywords: anemia, liver cirrhosis, portal hypertension, iron isomaltoside 


\section{Background}

Approximately $30 \%$ of the world's population is anemic, of which $50 \%$ is iron deficiency anemia (IDA) (1). Chronic liver diseases are frequently associated with hematological abnormalities. Anemia of diverse etiology occurs in about $75 \%$ of patients with chronic liver disease (2).

In some patients with cirrhosis, chronic hemorrhage into the gastrointestinal tract occurs. Esophageal and gastric varices and/or portal hypertensive gastropathy may be associated with slow chronic loss of blood into the gut and development of chronic IDA. Patients with severe hepatocellular disease develop defects of blood coagulation as a consequence of endothelial dysfunction, thrombocytopenia, deficiencies of coagulation factors and various associated disorders (3).

In liver cirrhosis, decreased synthesis of liver-produced plasma proteins leads to reduced serum levels of several blood clotting factors. Hemorrhage may occur as a complication of chronic liver disease because of a lack of one or more liver-produced blood clotting factors, thrombocytopenia, and/or defective platelet function. Hemorrhage in such patients may also occur from esophageal or gastric varices secondary to portal hypertension. Mucosal injury due to portal hypertension is an underestimated cause of occult bleeding and IDA (4).

Treatment with iron supplementation is indicated for IDA caused by chronic blood loss. In some cases of advanced chronic liver disease frequent blood transfusions may increase the risk of adverse events and toxicity due to development of antibodies against RBC as well as iron overload. Oral iron intake may be ineffective due to impaired absorption and may irritate the stomach.

Iron isomaltoside is a parenteral agent with low free-iron toxicity that is used as a short-term infusion without need of test-dose. Theoretically, this new drug seems to be very suitable for treatment of IDA in liver cirrhosis, but there are no safety data in this particular subgroup of patients so far.

\section{Case Description}

We present two clinical cases with IDA due to complications of liver cirrhosis who were successfully treated with iron isomaltoside.

Case number 1: a 30 year-old male was admitted to the Gastroenterology Clinic of St. Ivan Rilski University Hospital in 2014 with Child-A liver cirrhosis due to HCV infection and IDA. Liver cirrhosis was diagnosed in 2005. He received four courses of interferon-based therapy and sustained viral response was finally achieved with the last treatment course. There was a severe episode of bleeding from the upper Gl tract a year before admission. The patient had regular bowel habits with no clinical signs of bleeding. He experienced fatigue, and weakness. Physical examination showed signs of anemia and hepato-splenomegaly. The last was also confirmed by ultrasound examination.

Laboratory tests revealed anemia with complex etiology - IDA and hypersplenism (table 1).

Table 1: Laboratory tests showing iron-deficient anemia in patient 1

\begin{tabular}{|c|c|c|c|c|c|c|c|c|}
\hline Date & $\begin{array}{c}\text { Hb } \\
\mathbf{( g / L )}\end{array}$ & $\begin{array}{c}\text { RBC } \\
\text { (T/L) }\end{array}$ & Hct & $\begin{array}{c}\text { MCV } \\
(\mathbf{f L})\end{array}$ & $\begin{array}{c}\text { MCH } \\
\mathbf{( p g )}\end{array}$ & $\begin{array}{c}\text { Fe } \\
(\mathbf{u m o l} / \mathbf{l})\end{array}$ & $\begin{array}{c}\text { IBC } \\
(\mathbf{u m o l} / \mathbf{l})\end{array}$ & $\begin{array}{c}\text { PIt } \\
\mathbf{( G / I )}\end{array}$ \\
\hline 13.05 .14 & 83 & 4,0 & 0,27 & 67 & 20 & 3,2 & 82 & 60 \\
\hline
\end{tabular}




\begin{tabular}{|l|l|l|l|l|l|l|l|l|}
\hline 30.05 .14 & 80 & 4,1 & 0,26 & 64 & 19 & & & 63 \\
\hline
\end{tabular}

Fibrogastroscopy was performed and signs for severe portal hypertension were found - large oesophageal varices with red spots and portal hypertensive gastropathy without active bleeding.

Case number 2: A 66 year-old male was hospitalized in our department in 2014. Child-A alcoholic liver cirrhosis was diagnosed in 2008. IDA had been present since March 2014. He received several blood transfusions in the last year for correction of anemia and there was suspicion of auto red blood cell (RBC) antibodies. At hospital admission the patient had fatigue, weakness, and regular bowel habits with no clinical signs of bleeding. Physical examination and laboratory tests showed IDA (Table. 2)

Table 2. Laboratory tests showing iron-deficient anemia in patient 2

\begin{tabular}{|c|c|c|c|c|c|c|c|c|}
\hline Date & $\begin{array}{c}\mathbf{H b} \\
\mathbf{( g / L )}\end{array}$ & $\begin{array}{c}\mathbf{R B C} \\
\mathbf{( T / L})\end{array}$ & $\mathbf{H c t}$ & $\begin{array}{c}\mathbf{M C V} \\
\mathbf{( f L})\end{array}$ & $\begin{array}{c}\mathbf{M C H} \\
\mathbf{( p g})\end{array}$ & $\begin{array}{c}\mathbf{F e} \\
\mathbf{u m o l} / \mathbf{l})\end{array}$ & $\begin{array}{c}\text { IBC } \\
(\mathbf{u m o l} / \mathbf{l})\end{array}$ & $\begin{array}{c}\text { PIt } \\
\mathbf{( G / I )}\end{array}$ \\
\hline 25.06 .14 & 85 & 3,5 & 0,28 & 80 & 24 & 4,9 & 66 & 207 \\
\hline 03.07 .14 & 72 & 3,2 & 0,25 & 78 & 23 & & & 138 \\
\hline 21.08 .14 & 88 & 3,6 & 0,30 & 83 & 25 & 3,3 & 69 & 174 \\
\hline 01.10 .14 & 101 & 3,9 & 0,33 & 85 & 26 & 6,2 & 61 & 180 \\
\hline 27.11 .14 & 100 & 3,9 & 0,32 & 82 & 26 & 7,0 & 67 & 213 \\
\hline 20.02 .15 & 65 & 3,2 & 0,23 & 71 & 20 & 2,8 & 75 & 178 \\
\hline 04.03 .15 & 89 & 3,9 & 0,31 & 79 & 23 & 189 & & \\
\hline
\end{tabular}

Fibrogastroscopy revealed severe portal hypertension - large oesophageal varices without red signs and portal hypertensive gastropathy without active bleeding. Fibrocolonoscopy was also performed and portal colopathy as well as diverticulosis of the sigma were found. No active bleeding was seen.

This subject was indicated for treatment of anemia in order to:

- ameliorate $\mathrm{Hb}$ levels (and/or normalize them if it's possible) and improve patient's performance

- perform diagnostic endoscopy in a safe manner

- prevent the formation of auto red blood cell (RBC) antibodies by avoiding further blood transfusions

- prevent hepatic injury caused by iron overload that may result after frequent blood transfusions especially in case of development of anti RBC antibodies 


\section{Results}

Anemia has been previously orally-treated with ferric-hydroxide polymaltose complex, however no improvement was achieved.

Both patients were treated with iron isomaltoside infusions.

1) The younger one received a single dose of $1000 \mathrm{mg}$ and after one month $\mathrm{Hb}$ levels increased from 80 to $122 \mathrm{~g} / \mathrm{l}$.

2) The older subject was treated initially with $600 \mathrm{mg}$. As a result, $\mathrm{Hb}$ levels remained relatively unchanged.

A second infusion in dose of $1200 \mathrm{mg}$ was given one-month later and $\mathrm{Hb}$ levels increased from 72 to 88 $\mathrm{g} / \mathrm{l}$ (table 2). The ferritin levels increased from 14 to $23 \mathrm{ng} / \mathrm{mL}$ (normal range in males: $12-300 \mathrm{ng} / \mathrm{mL}$, female: $12-150 \mathrm{ng} / \mathrm{mL}$ ). Hemoglobin levels decreased between treatment cycles with iron. Another series of three infusions of iron was administered to the older patient in a dose of $1000 \mathrm{mg}$ monthly. Before the fifth iron infusion ferritin levels were $8 \mathrm{ng} / \mathrm{mL}$. As a result of the fifth iron infusion, Hb levels increased from 65 to $89 \mathrm{~g} / \mathrm{l}$ (table 2).

Both subjects were with normal liver enzymes prior to iron isomaltoside infusions and remained the same after the therapy (Table 3 and 4).

Table 3. Liver enzymes before iron isomaltoside infusion

\begin{tabular}{|l|c|c|c|c|}
\hline & ASAT & ALAT & GGT & ALP \\
\hline Patient (case 2) & 20 & 13 & 61 & 60 \\
\hline Patient (case 1) & 18 & 21 & 30 & 40 \\
\hline
\end{tabular}


Table 4. Liver enzymes after iron isomaltoside infusion

\begin{tabular}{|c|c|c|c|c|}
\hline & ASAT & ALAT & GGT & ALP \\
\hline $\begin{array}{c}\text { Patient (case 2) } \\
\text { After the first iron infusion }\end{array}$ & 20 & 13 & 61 & 60 \\
\hline$-\quad$ After the second iron infusion & 17 & 9 & 51 & 57 \\
\hline$-\quad$ After the third iron infusion & 17 & 10 & 50 & 50 \\
\hline After the fourth iron infusion & 20 & 13 & 54 & 51 \\
\hline After the fifth iron infusion & 19 & 10 & 54 & 61 \\
\hline After the sixth iron infusion & 15 & 12 & 54 & 60 \\
& & & & \\
\hline Patient (case 1) & 27 & 39 & 30 & 43 \\
& & & & \\
\hline
\end{tabular}

Treatment was well tolerated and no side effects were observed.

\section{Discussion}

Liver diseases are frequently associated with hematological abnormalities. Anemia of diverse etiology occurs in many of these patients. Occult bleeding is one of the most severe causes of anemia in patients with liver cirrhosis complicated with portal hypertension, especially if portal gastropathy or colonopathy exists together with coagulation abnormalities.

Alcohol is implicated in the pathogenesis of chronic liver disease and may also contribute to anemia secondary to its direct effects on the liver. Folic acid and vitamin B12 deficiencies develop frequently in alcoholics. These deficiencies may be related to inadequate food intake or intestinal malabsorption. Moreover, anemia may also result as a consequence of the direct toxic effects of alcohol on erythrocyte precursors in the bone marrow as well as from antifolate action of ethanol (5). 
As a consequence of anemia, cirrhotic patients experienced pronounced weakness and reduced daily performance. Iron is essential for oxygen transport and since the liver is a metabolically important organ and also a major iron-storing organ, a variety of hepatic metabolic changes may occur in case of iron deficiency. In animal models IDA caused impaired lipid metabolism with increased serum triacylglycerol (TG), phospholipids, low-density lipoprotein (LDL), and very low-density lipoprotein (VLDL) cholesterol in the serum, as well as elevation of TG and phospholipids in the liver (6). Iron deficiency may also result in the expression of genes encoding gluconeogenic enzymes and thus increase serum glucose levels and insulin levels, so insulin resistance may occur. In iron-deficient setting the serum levels of amino acids-free alanine, phenylalanine, lysine, leucine, isoleucine, methionine, proline, asparagine, and taurine increased, while those of free citrulline and threonine decreased. The level of ammonia also increased. And finally, it was demonstrated that IDA simultaneously influences not only nutrient metabolism, but even apoptosis as a consequence of endoplasmic reticulum stress in the liver (6).

The above data clearly suggest that compensation of IDA in cirrhosis is an important therapeutic step for improvement of liver metabolism. This treatment should be carefully performed in order to avoid iron overload as it may aggravate liver disease activity as well as progression of fibrosis.

This is the first report for treatment of iron-deficiency anemia by iron isomaltoside infusion in cirrhotic patients.

The new IV iron, iron isomaltoside 1000, which has recently become commercially available in several European countries, is composed of iron and chemically modified isomalto- oligosaccharides which have a mean molecular weight of $1000 \mathrm{Da}$ and consist predominantly of 3-5 glucose units. In contrast to dextrans, the carbohydrate isomaltoside 1000 is a linear and unbranched structure with a theoretically low immunological potential. Iron isomaltoside 1000 contains strongly bound iron within the iron-isomaltoside formulation, which enables a controlled, slow release of bioavailable iron to the iron-binding proteins, with potentially a reduced risk of free iron toxicity. In practice there is no likelihood of hepatotoxicity as the possibility to use a single controlled infusion precludes iron overload.

In these two cases iron isomaltoside has proved to be effective by improving hemoglobin levels and has improved the patients' performance. No side effects occurred after intravenous application. Hepatotoxicity because of the iron overload was not observed. The throwaway controlled import precludes iron overload..

\section{Conclusion}

Iron isomaltoside infusion may be a good option of anemia therapy when blood transfusion is contraindicated and with risk.

Further studies are needed to establish its safety and efficacy in subjects with liver cirrhosis.

\section{References}

1. Yakub I, Naresh T. Gunaratnam, Nonvariceal Upper Gastrointestinal bleeding. Clinical Gastrointestinal Endoscopy 2012;33:567-670.

2. McHutchison JG, Manns MP, Longo DL. Definition and management of anemia in patients infected with hepatitis C virus. Liver Int 2006;26:389-398.

3. Caldwell SH, Hoffman M, Lisman T, et al. Coagulation disorders and hemostasis in liver disease: pathophysiology and critical assessment of current management. Hepatology 2006;44:10391046. 
4. Charanjit V, Coelho-Prabhu N, Navtej SB. Portal hypertensive bleeding. Clinical Gastrointestinal Endoscopy 2012;45:167-167.

5. Gonzalez-Casas R, Jones EA, Moreno-Otero R. Spectrum of anemia associated with chronic liver disease. World J Gastroenterol 2009;15:37:4653-4658.

6. Kamei A, Watanabe $Y$, Ishijima $T$, et al. Dietary iron-deficient anemia induces a variety of metabolic changes and even apoptosis in rat liver: a DNA microarray study. Physiol Genomics 2010;42:149-156.

\section{Corresponding author}

Liuben Milatchkov

Adres: 15 Akad. Ivan Geshov Blvd., Sofia 1431, Bulgaria,

University Hospital „St. Ivan Rilski”, Clinic of Gastroenterology

E-mail: lub26@hotmail.com

fax number: +35928510615 